\title{
Rapid Progression of Liver Fibrosis Induced by Acute Liver Injury Due to Immune-related Adverse Events of Atezolizumab
}

\author{
Yuichi Honma ${ }^{1}$, Michihiko Shibata ${ }^{1}$, Tomonori Gohda ${ }^{1}$, Hiroki Matsumiya ${ }^{2}$, \\ Keiichiro Kumamoto ${ }^{1,3}$, Aya Miyama ${ }^{1}$, Kahori Morino ${ }^{1}$, Yudai Koya ${ }^{1,4}$, Akihiro Taira ${ }^{2}$, \\ Shinji Shinohara ${ }^{2}$, Tsuguru Hayashi ${ }^{1}$, Masashi Kusanaga ${ }^{1}$, Shinji Oe ${ }^{1}$, Koichiro Miyagawa ${ }^{1}$, \\ Shintaro Abe ${ }^{1}$, Fumihiro Tanaka ${ }^{2}$ and Masaru Harada ${ }^{1}$
}

\begin{abstract}
:
A 72-year-old woman with advanced lung cancer had received systemic chemotherapy including atezolizumab. About three months after the initial administration of atezolizumab, her liver enzyme levels increased. The histopathological findings of the initial liver biopsy revealed acute inflammatory infiltrate, predominantly $\mathrm{CD}^{+}, \mathrm{CD}^{+}$and $\mathrm{CD}^{+} \mathrm{T}$ lymphocytes, in the hepatic lobules. We diagnosed her with atezolizumab-induced immune-related acute hepatitis. Oral corticosteroid therapy successfully improved the elevation of serum aminotransferases. A sequential liver biopsy demonstrated the rapid progression of liver fibrosis. Because hepatocellular carcinoma occurs most often in advanced cases of chronic liver disease, we should pay close attention to immune-related acute hepatic injury when treating patients with advanced liver diseases using atezolizumab.
\end{abstract}

Key words: atezolizumab, autoimmune hepatitis, immune checkpoint inhibitor, immune-related adverse event, sequential liver biopsy, liver fibrosis

(Intern Med 60: 1847-1853, 2021)

(DOI: 10.2169/internalmedicine.6535-20)

\section{Introduction}

Immune checkpoint inhibitors (ICIs) are monoclonal antibodies targeting immune checkpoint molecules. ICIs target three main molecules: including programmed cell death receptor-1 (PD-1), programmed cell death ligand-1 (PD-L1), and cytotoxic T-lymphocyte-associated molecule-4 (CTLA4). ICIs trigger an immune-mediated anti-tumor response by promoting the activation of cytotoxic $\mathrm{T}$ lymphocytes. ICIs have led to important clinical breakthroughs in the field of immunotherapy for the treatment of advanced malignancies and improved the overall survival (1).

Recently, the combination of atezolizumab, a humanized monoclonal immunoglobulin (Ig) G1 antibody to the PD-L1, and bevacizumab, an anti-vascular endothelial growth factor (VEGF) antibody, has demonstrated efficacy and been adapted for the treatment of hepatocellular carcinoma (HCC) (2). Although ICIs have been proven to be highly effective for various malignancies, they sometimes induce significant immune-related adverse events (irAEs), which mimic autoimmune diseases, including hypothyroidism, adrenal insufficiency, thyroiditis, diabetes, enterocolitis and hepatitis. The mechanisms underlying the development of immune-related hepatitis have been presumed to be similar to those of autoimmune hepatitis (AIH) (3). However, the clinical features of immune-related hepatic injury induced by ICIs have yet to be fully clarified.

\footnotetext{
${ }^{1}$ Third Department of Internal Medicine, School of Medicine, University of Occupational and Environmental Health, Japan, ${ }^{2}$ Second Department of Surgery, School of Medicine, University of Occupational and Environmental Health, Japan, ${ }^{3}$ Department of Pathology, School of Medicine, University of Occupational and Environmental Health, Japan and ${ }^{4}$ Department of Gastroenterology, Moji Medical Center, Japan Received: October 16, 2020; Accepted: November 29, 2020; Advance Publication by J-STAGE: January 15, 2021 Correspondence to Dr. Yuichi Honma, y-homma@med.uoeh-u.ac.jp
} 

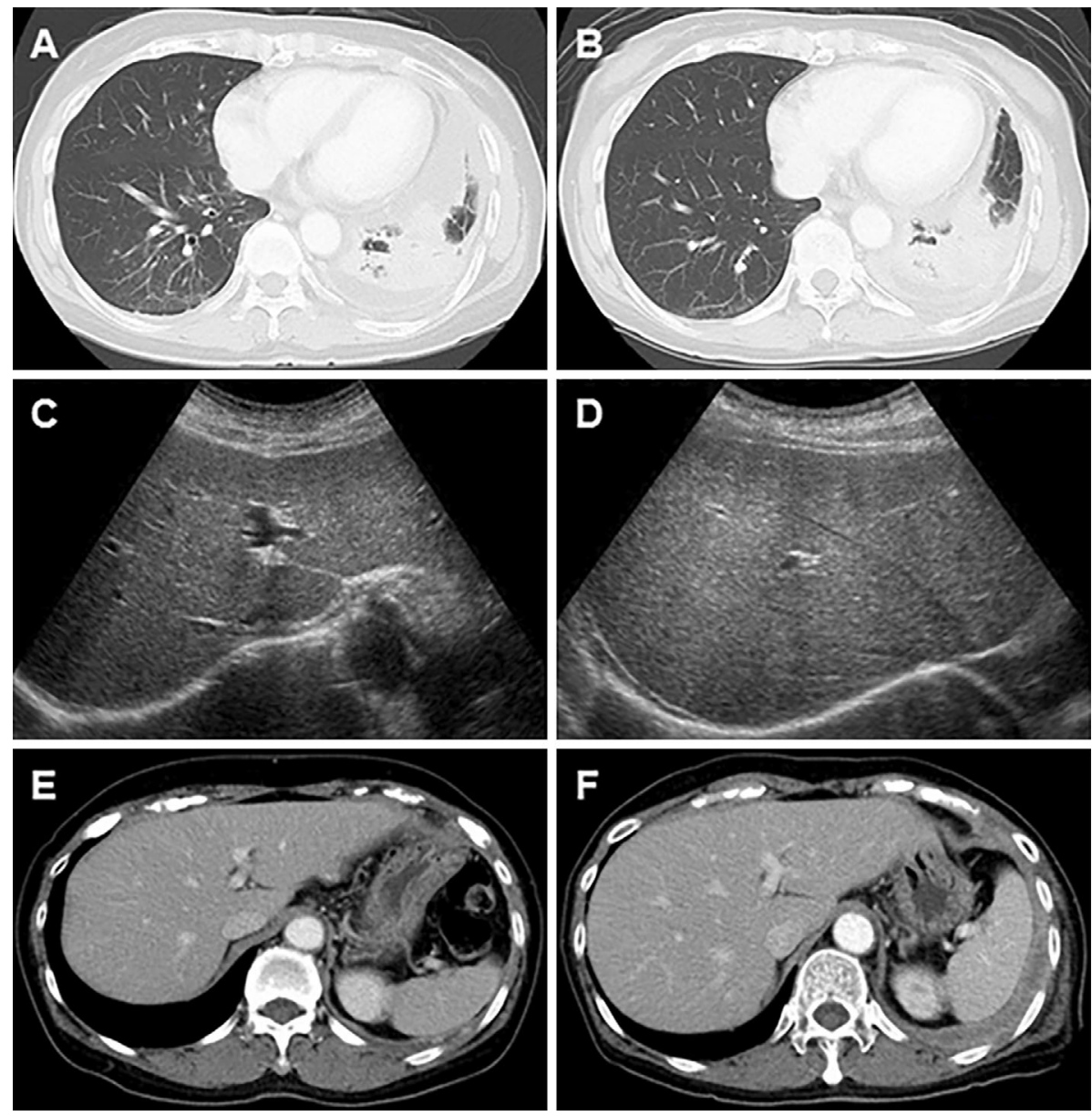

Figure 1. Imaging findings of computed tomography (CT) and abdominal ultrasonography of this patient. Chest CT demonstrated no marked changes in lung cancer by comparing the findings pretreatment (A) and after three courses administration of atezolizumab in combination with chemotherapy (B). Abdominal ultrasonography (C, D) and contrast-enhanced-CT (F) on admission showed no liver metastasis of lung cancer. Hepatomegaly was evident on the comparison of $\mathrm{CT}$ findings at pre-treatment (E) and admission (F).

We herein report a Japanese patient with advanced nonsmall-cell lung cancer who developed acute hepatitis during treatment with the combination of atezolizumab plus bevacizumab, carboplatin and paclitaxel. Although corticosteroid therapy immediately improved the liver injury, comparing the histopathological findings between the liver biopsy at the diagnosis and two month later revealed the rapid progression of liver fibrosis.

\section{Case Report}

A 72-year-old Japanese woman was referred to our hospital due to left chest pain. She was diagnosed with nonsmall-cell lung cancer with pleural dissemination by a thoracoscopic biopsy (Fig. 1A). She had no history of acute or chronic liver diseases. She had never smoked and did not have a history of habitual alcohol consumption. There was no family history of liver diseases. Her body weight was $45.4 \mathrm{~kg}$, and her height was $148.8 \mathrm{~cm}$, giving a body mass index of $20.5 \mathrm{~kg} / \mathrm{m}^{2}$. She started intravenous atezolizumab (1,200 mg/body) in combination with bevacizumab $(15 \mathrm{mg} /$ $\mathrm{kg}$ body weight), carboplatin and paclitaxel every four weeks as first-line systemic chemotherapy, receiving three courses of this regimen for three months. This regimen effectively controlled her advanced lung cancer with a Response Evaluation Criteria in Solid Tumors (RECIST) classification of stable disease (Fig. 1B), and no adverse events were observed after three courses chemotherapy.

However, at three months after the initiation of this chemotherapy, she developed liver injury. We stopped the administration of the combination of atezolizumab and other anti-cancer drugs and started treatment of ursodeoxycholic acid (UDCA) (300 mg/day). Because her liver injury gradually worsened, she was admitted to our department. A physical examination showed no remarkable abnormality. A laboratory examination (Table) showed elevated aspartate aminotransferase (AST), alanine aminotransferase (ALT), alkaline phosphatase (ALP) and gamma-glutamyl transpeptidase $(\gamma-\mathrm{GTP})$. Hepatitis B virus (HBV) DNA and hepatitis C virus (HCV) antibody were negative. Antinuclear antibody (ANA) was positive (1:40) with a speckled pattern and slightly elevated IgG. On abdominal ultrasonography (US) 
Table. Characteristics of This Patient on Admission.

\begin{tabular}{|c|c|c|c|}
\hline Hematology & & Serology & \\
\hline WBC & $7.0 \times 10^{3} / \mu \mathrm{L}$ & CRP & $3.5 \mathrm{mg} / \mathrm{dL}$ \\
\hline Neut & $55.9 \%$ & & \\
\hline Eos & $4.3 \%$ & Coagulation & \\
\hline $\mathrm{RBC}$ & $3.4 \times 10^{6} / \mu \mathrm{L}$ & $\mathrm{PT}$ & $14.1 \mathrm{sec}$ \\
\hline $\mathrm{Hb}$ & $9.4 \mathrm{~g} / \mathrm{dL}$ & PT-\% & $75 \%$ \\
\hline Hct & $29.3 \%$ & PT-INR & 1.12 \\
\hline \multirow[t]{2}{*}{ Plt } & $33.3 \times 10^{3} / \mu \mathrm{L}$ & & \\
\hline & & Immunology & \\
\hline Biochemistry & & ANA & $80 x$ \\
\hline ТP & $7.1 \mathrm{~g} / \mathrm{dL}$ & Homogeneous & $(-)$ \\
\hline Alb & $3.2 \mathrm{~g} / \mathrm{dL}$ & Speckled & $(+)$ \\
\hline T-Bil & $0.6 \mathrm{mg} / \mathrm{dL}$ & Centromere & $(-)$ \\
\hline D-Bil & $0.2 \mathrm{mg} / \mathrm{dL}$ & Nucleolar & $(-)$ \\
\hline AST & $313 \mathrm{U} / \mathrm{L}$ & Peripheral & $(-)$ \\
\hline ALT & $208 \mathrm{U} / \mathrm{L}$ & Granular & $(-)$ \\
\hline LDH & $295 \mathrm{U} / \mathrm{L}$ & Anti-LKM1 & $<5$ index \\
\hline ALP & $1,075 \mathrm{U} / \mathrm{L}$ & AMA & $<20 x$ \\
\hline$\gamma$-GTP & $285 \mathrm{U} / \mathrm{L}$ & AMA M2 & 1.4 \\
\hline BUN & $11 \mathrm{mg} / \mathrm{dL}$ & $\mathrm{IgG}$ & $1,870 \mathrm{mg} / \mathrm{dL}$ \\
\hline Cre & $0.57 \mathrm{mg} / \mathrm{dL}$ & $\operatorname{IgM}$ & $111 \mathrm{mg} / \mathrm{dL}$ \\
\hline $\mathrm{Na}$ & $140 \mathrm{mmol} / \mathrm{L}$ & IgG4 & $82.1 \mathrm{mg} / \mathrm{dL}$ \\
\hline K & $4.1 \mathrm{mmol} / \mathrm{L}$ & & \\
\hline $\mathrm{Cl}$ & $102 \mathrm{mmol} / \mathrm{L}$ & Tumor markers & \\
\hline $\mathrm{ChE}$ & $236 \mathrm{U} / \mathrm{L}$ & AFP & $4.6 \mathrm{ng} / \mathrm{mL}$ \\
\hline CPK & $36 \mathrm{U} / \mathrm{L}$ & CEA & $2.4 \mathrm{ng} / \mathrm{mL}$ \\
\hline $\mathrm{Fe}$ & $30 \mu \mathrm{g} / \mathrm{dL}$ & SLX & $32.3 \mathrm{U} / \mathrm{mL}$ \\
\hline Ferritin & $701 \mathrm{ng} / \mathrm{mL}$ & & \\
\hline TSH & $1.77 \mu \mathrm{IU} / \mathrm{mL}$ & Virus markers & \\
\hline FT3 & $2.5 \mathrm{pg} / \mathrm{mL}$ & HBs Ag & $(-)$ \\
\hline FT4 & $1.19 \mathrm{ng} / \mathrm{dL}$ & $\mathrm{HBs} \mathrm{Ab}$ & $(+)$ \\
\hline FPG & $78 \mathrm{mg} / \mathrm{dL}$ & $\mathrm{HBc} \mathrm{Ab}$ & $(+)$ \\
\hline $\mathrm{HbA1c}$ & $5.6 \%$ & HBV DNA & $(-)$ \\
\hline Hyaluronic acid & $62 \mathrm{ng} / \mathrm{mL}$ & $\mathrm{HCV} \mathrm{Ab}$ & $(-)$ \\
\hline Type IV collagen & $134 \mathrm{ng} / \mathrm{mL}$ & & \\
\hline M2BPGi & $2.29 \mathrm{COI}$ & Urinalysis & \\
\hline Autotaxin & $0.727 \mathrm{mg} / \mathrm{L}$ & Protein & $( \pm)$ \\
\hline
\end{tabular}

TP: total protein, Alb: albumin, T-Bil: total bilirubin, D-Bil: direct bilirubin, Ig: immunoglobulin, AST: aspartate aminotransferase, ALT: alanine aminotransferase, LDH: lactate dehydorogenase, ALP: alkaline phosphatase, $\gamma$-GTP: gamma-glutamyl transpeptidase, BUN: blood urea nitrogen, Cre: creatinine, ChE: cholinesterase, CPK: creatine phosphokinase, TSH: thyroid-stimulating hormone, FPG: fast plasma glucose, M2BPGi: Mac2-binding protein glycosylation isomer, CRP: C-reactive protein, PT: prothrombin time, ANA: antinuclear antibody, LKM: liver kidney microsome, AMA: antimitochondrial antibody, Ig: immunoglobulin, AFP: alfa fetoprotein, CEA: carcinoembryonic antigen, SLX: Sialyl Lewis X, HBV: hepatitis B virus, HCV: hepatitis C virus

(Fig. 1C, D) and contrast-enhanced computed tomography (CT) (Fig. 1F) performed at admission, no liver metastasis was evident. CT demonstrated a swollen liver (Fig. 1F) compared to the findings before chemotherapy (Fig. 1E). Periportal edema or periportal color sign were not evident. The histopathological findings of the first liver biopsy revealed moderately acute inflammatory infiltrate and spotty necrosis within hepatic lobules and few plasma cells in the portal area with disrupted limiting plates (Fig. 2A), whereas liver fibrosis was limited (Fig. 2B). Immunostaining for im- mune cell infiltration showed that $\mathrm{CD}^{+}, \mathrm{CD}^{+}$and $\mathrm{CD}^{+} \mathrm{T}$ lymphocytes were predominantly observed (Fig. 3A-C), while $\mathrm{CD} 20^{+}$B lymphocytes were rare (Fig. 3D). We diagnosed her with immune-related acute hepatitis due to atezolizumab.

Although we recommended starting corticosteroid therapy immediately, she rejected the idea due to adverse effects. One month later, however, she accepted our proposal, and we started the oral administration of prednisolone $45 \mathrm{mg} /$ day $(1 \mathrm{mg} / \mathrm{kg} /$ day $)$ and increased dose of UDCA $(600 \mathrm{mg} /$ 

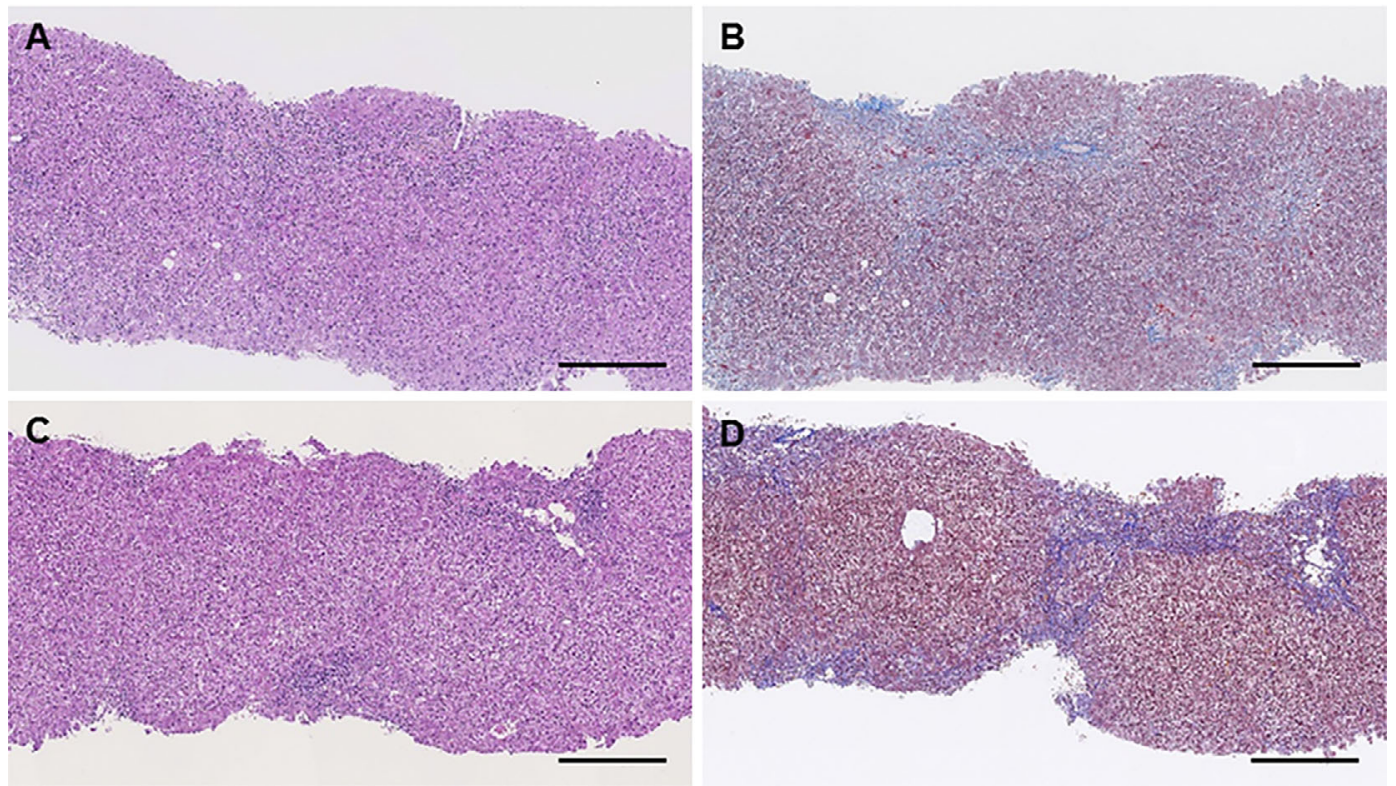

Figure 2. Histopathological findings of the liver biopsy specimen. The first liver biopsy (A, B) showed active pan-lobular hepatitis with moderate inflammatory infiltrate consisting of lymphocytes, macrophages and a few plasma cells in the portal area with disrupted limiting plates and spotty necroses within hepatic lobules. Scattered eosinophils were also seen. Liver fibrosis was limited. The second liver biopsy (C, D) demonstrated mild to moderate inflammatory infiltrate and fibrosis with bridging fibrosis associated with scattered intralobular spotty or focal necrosis. (A), (C) Hematoxylin and Eosin staining (×100). (B), (D) Masson-trichrome staining $(\times 100)$. Scale bars: $500 \mu \mathrm{m}$.

day). Subsequently, the elevation of both serum aminotransferases and IgG immediately improved. ALP and $\gamma$-GTP were also improved after the administration of prednisolone (Fig. 4). The total bilirubin level increased to $3.2 \mathrm{mg} / \mathrm{dL}$, and the prothrombin time (PT) decreased to $60.7 \%$ after the initiation of the chemotherapy. Corticosteroid therapy immediately improved both the total bilirubin and prothrombin time. Because no recurrence in the serum aminotransferase elevation was observed, we tapered the prednisolone dose.

One month after the initiation of corticosteroid therapy, we performed a second liver biopsy. This liver biopsy showed that the inflammatory infiltrate in hematoxylin and eosin staining (Fig. 2C) and immunostaining of $\mathrm{CD}^{+}, \mathrm{CD}^{+}$ and $\mathrm{CD}^{+} \mathrm{T}$ lymphocytes (Fig. 3E-G) had improved. Infiltrate of $\mathrm{CD}_{2} 0^{+}$was rare (Fig. $3 \mathrm{H}$ ). In contrast, the liver fibrosis rapidly progressed, resulting in bridging fibrosis (Fig. 2D). Serum liver fibrosis markers, including hyaluronic acid (62 vs. 1,260 ng/mL), type 4 collagen (134 vs. $186 \mathrm{ng}$ / $\mathrm{mL}$ ), Mac-2-binding protein glycosylation isomer (M2BPGi) (2.29 vs. $3.15 \mathrm{COI})$ and autotaxin (0.72 vs. $0.94 \mathrm{mg} / \mathrm{L})$, were higher at the second liver biopsy than at the first liver biopsy.

\section{Discussion}

Immune-related hepatitis induced by ICIs seems to be an infrequent but well-known irAE. It is often seen between 6 and 14 weeks following the initiation of ICIs but may occur at any time (4). The incidence of any-grade (grade 3-4) liver injury was reported to be $1-10 \%(1-2 \%)$ in patients receiving an approved dose of anti-PD-1 and anti-CTLA-4 monotherapy $(5,6)$. In some cases, ICIs can lead to acute liver failure $(7,8)$.

Atezolizumab (Tecentriq ${ }^{\circledR}$ ) is an anti-PD-L1 antibody initially approved for non-small-cell lung cancer and urothelial carcinoma by the Food and Drug Administration (FDA) in 2016. Liver injury was shown to occur in $8-10 \%$ (grade 3-4 in $1-2 \%$ ) of patients in the clinical trials of atezolizumab in combination with chemotherapy in advanced lung cancer $(9,10)$. The safety profile of atezolizumab in combination with anti-cancer agents was consistent with the known adverse events related to single-agent atezolizumab, and no new adverse events were observed (9). A phase 3 clinical study revealed benefit to the overall and progression-free survival rates by the combination of atezolizumab and bevacizumab in unresectable HCC (11). Following the results of this study, atezolizumab became the first clinically approved ICI in the treatment of advanced HCC. The incidence of any-grade (grade 3-4) increases in AST and ALT in this clinical trial was $19.0 \%$ and $14.0 \%$ (7.0\% and $3.6 \%$ ), respectively (11). The incidence of liver injury in HCC therefore seems to be more frequent than in other cancers. Treatment of bevacizumab in combination with anti-cancer agents except for ICIs has not been considered to be associated with liver injury (12). The incidence of elevation of aminotransferases in bevacizumab therapy for advanced HCC was shown to be $38 \%$ (grade 1-2) and $13 \%$ (grade 34) (13). VEGF has been reported to have immune- 

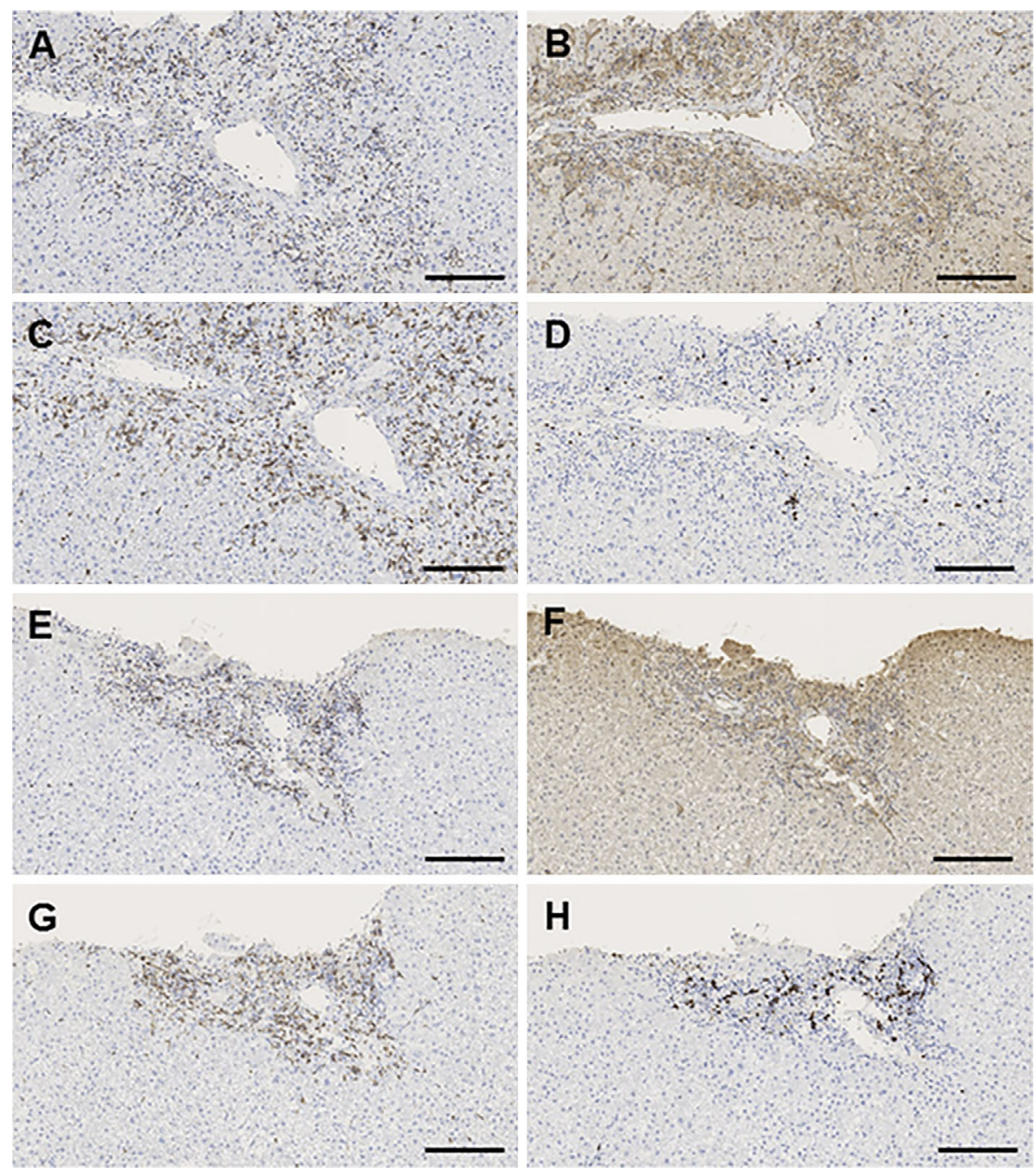

\section{Figure 3. Immunostaining of $\mathrm{CD3}^{+}(\mathrm{A}), \mathrm{CD4}^{+}\left(\mathrm{B}^{2}, \mathrm{CD8}^{+}(\mathrm{C})\right.$ and $\mathrm{CD20}^{+}(\mathrm{D})$ of the first liver biopsy $(\times 200)$ and $\mathrm{CD3}^{+}(\mathrm{E}), \mathrm{CD4}^{+}(\mathrm{F}), \mathrm{CD8}^{+}(\mathrm{G})$ and $\mathrm{CD20}^{+}(\mathrm{H})$ of the second liver biopsy $(\times 200)$. Scale bars: $200 \mu \mathrm{m}$.}

suppressive effects, such as downregulating $\mathrm{T}$ cell activation and increasing myeloid-derived suppressor cells and regulatory $\mathrm{T}$ cells in the tumor microenvironment (14). Because anti-VEGF treatment is considered to enhance the immunomodulating effects of anti-PD-L1 therapy, the combination of atezolizumab plus bevacizumab may increase irAEs, including immune-related hepatic injury.

$\mathrm{AIH}$ is an immune-mediated inflammatory liver disease characterized by autoantibodies and hypergammaglobulinemia. In contrast, immune-related hepatitis due to ICIs apparently has immune-mediated mechanisms, although most patients do not develop conventional autoantibodies. The ANA titer and extent of IgG elevation in immune-related hepatitis was reported to be lower than in classical AIH (15). In the present case, ANA was mildly positive with a low titer (1: 40-1:80), and serum IgG was slightly elevated.

The histopathological features of immune-related hepatitis have been reported, with anti-CTLA-4 inducing active panlobular hepatitis with inflammatory infiltrate consisting predominantly of lymphocytes and anti-PD-1 exhibiting lobular hepatitis with mild portal inflammation and scattered necro- sis (16). In the present patient, the histopathological findings revealed acute inflammatory infiltrate within the hepatic lobules and interface hepatitis, but plasma cells were few. These findings were compatible with the previously reported immune-related hepatitis (17). We also evaluated the immune cell infiltration by immunostaining including $\mathrm{T}$ lymphocytes $\left(\mathrm{CD}^{+}\right)$, cytotoxic $\mathrm{T}$ lymphocytes $\left(\mathrm{CD} 8^{+}\right)$, helper $\mathrm{T}$ lymphocytes $\left(\mathrm{CD}^{+}\right)$and $\mathrm{B}$ lymphocytes $\left(\mathrm{CD} 20^{+}\right) . \mathrm{CD}^{+}, \mathrm{CD}$ $4^{+}$and $\mathrm{CD}^{+}$were predominantly observed, as previously reported (18). Serum bilirubin and the biliary enzymes ALP and $\gamma$-GTP were also elevated in this case. We previously reported secondary sclerosing cholangitis due to an irAE by pembrolizumab (19). However, neither bile duct dilation on abdominal US or CT nor histological cholestatic changes on a liver biopsy were detected.

Immune-related hepatitis usually resolves within four to six weeks after stopping ICIs but is occasionally prolonged. The effectiveness of corticosteroid therapy has been demonstrated (20). Corticosteroid therapy can allow the continuation of treatment with ICIs, but rare cases have been reported to be refractory to steroids (21). In our case, corti- 


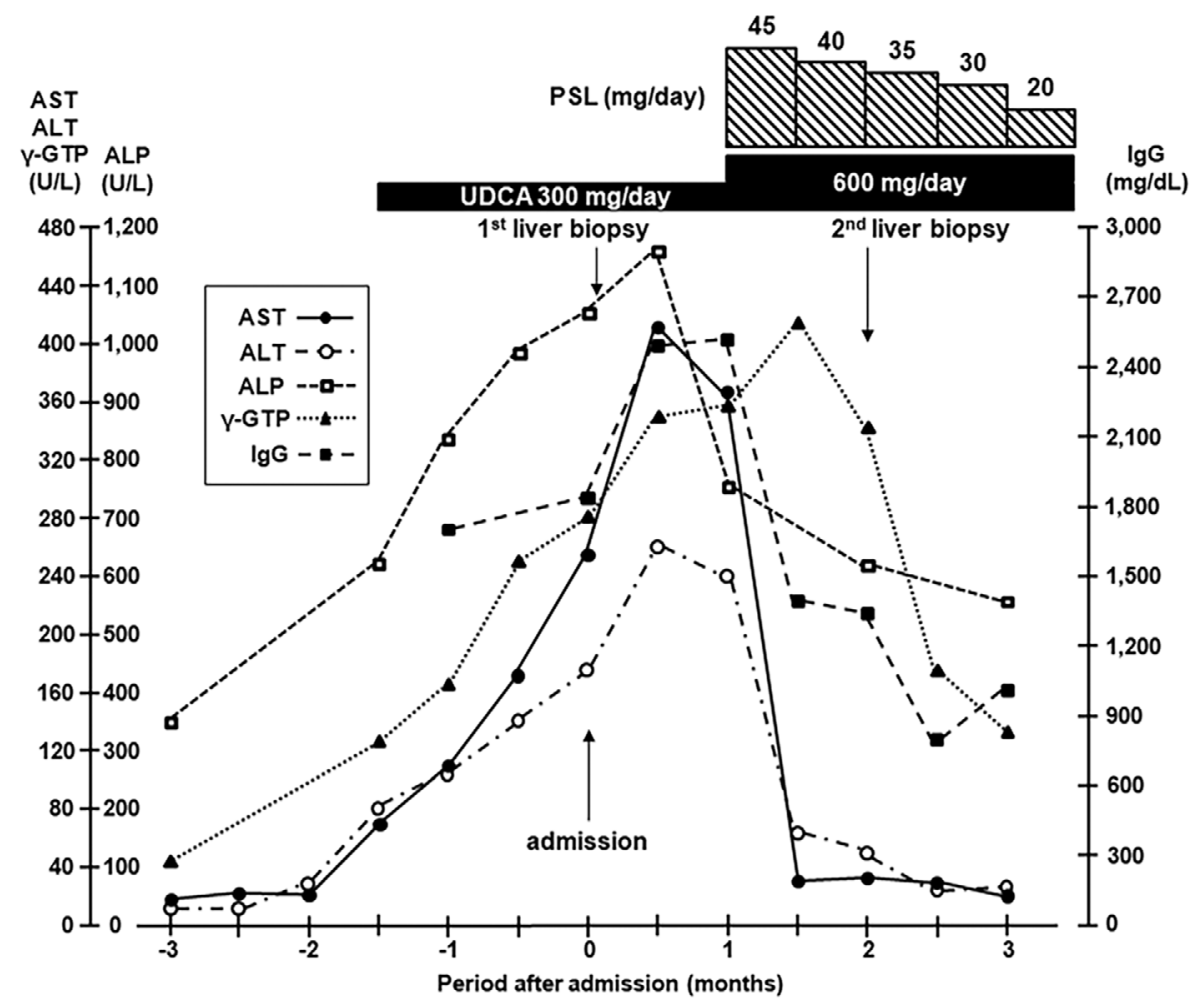

Figure 4. Clinical course of the present case. Treatment with $300 \mathrm{mg} / \mathrm{day}$ ursodeoxycholic acid (UDCA) was started at $\mathbf{1 . 5}$ months before the first liver biopsy. Because the liver injury worsened, the first liver biopsy was performed for the histopathological diagnosis. One month later, oral prednisolone (PSL) $45 \mathrm{mg} / \mathrm{day}$ (1 mg/kg/day) was started, and the dose of UDCA was increased (600 mg/day). After starting PSL, the elevation of serum aspartate aminotransferase (AST), alanine aminotransferase (ALT) and immunoglobulin (Ig) G immediately improved. Serum alkaline phosphatase (ALP) and gamma-glutamyl transpeptidase ( $\gamma$-GTP) levels also gradually decreased. Subsequently, oral PSL was tapered, and no recurrence in the elevation of serum AST, ALT, ALP or $\gamma$-GTP levels was observed. A second liver biopsy was performed one month after starting treatment with PSL.

costeroid therapy successfully improved the elevation of serum aminotransferases and $\mathrm{IgG}$. Furthermore, the improvement in the histopathological findings of $\mathrm{CD}^{+}, \mathrm{CD}^{+}$and $\mathrm{CD}^{+}$lymphocyte infiltration was demonstrated at the second liver biopsy performed one month after the initiation of corticosteroid therapy. Because atezolizumab and bevacizumab controlled the lung cancer in this patient well, we considered continuation of this combination with corticosteroid therapy favorable for this patient. Surprisingly, the second liver biopsy performed two months after the first one revealed the rapid progression of the liver fibrosis during this short term. Rapid progression to acute liver failure or hepatic decompensation has been demonstrated in some cases with AIH (22). However, a previous report suggested that immune-related hepatitis could induce liver fibrosis $(17,23)$. To our knowledge, few reports have described the progression of liver fibrosis due to immune-related acute hepatitis by sequential paired liver biopsies, except for two case reports involving treatment with nivolumab (23) and pembrolizumab (17). Because immune-related hepatitis can start off as asymptomatic or with minimal symptoms (most commonly elevation of AST and ALT with mild elevation of total bilirubin), a histopathological assessment by a liver bi- opsy seems necessary in addition to careful monitoring of liver enzymes. Serum liver fibrosis markers, such as hyaluronic acid, type 4 collagen, M2BPGi and autotaxin are also helpful for assessing the progression of liver fibrosis.

In conclusion, immune-related hepatitis due to atezolizumab can induce rapid liver fibrosis progression despite improvements in the liver injury by corticosteroid therapy. Even in cases of immune-related acute hepatitis, a liver biopsy should be considered for evaluating the severity of liver fibrosis. HCC occurs most often in advanced chronic liver diseases, such as liver cirrhosis. We should therefore pay closer attention to immune-related hepatic injury during the treatment of HCC using ICIs than during treatment of other malignancies.

The authors state that they have no Conflict of Interest (COI).

\section{References}

1. Sharma P, Allison JP. The future of immune checkpoint therapy. Science 348: 56-61, 2015.

2. Hack SP, Spahn J, Chen M, et al. IMbrave 050: a phase III trial of atezolizumab plus bevacizumab in high-risk hepatocellular carci- 
noma after curative resection or ablation. Future Oncol 16: 975989, 2020.

3. Johncilla M, Misdraji J, Pratt DS, et al. Ipilimumab-associated hepatitis: clinicopathologic characterization in a series of 11 cases. Am J Surg Pathol 39: 1075-1084, 2015.

4. Spain L, Diem S, Larkin J. Management of toxicities of immune checkpoint inhibitors. Cancer Treat Rev 44: 51-60, 2016.

5. Wolchok JD, Chiarion-Sileni V, Gonzalez R, et al. Overall survival with combined nivolumab and ipilimumab in advanced melanoma. N Engl J Med 377: 1345-1356, 2017.

6. Haanen JBAG, Carbonnel F, Robert C, et al.; ESMO Guidelines Committee. Management of toxicities from immunotherapy: ESMO clinical practice guidelines for diagnosis, treatment and follow-up. Ann Oncol 28 (Suppl 4): iv119-iv142, 2017.

7. Imafuku K, Yoshino K, Yamaguchi K, Tsuboi S, Ohara K, Hata H. Successful treatment of sudden hepatitis induced by long-term nivolumab administration. Case Rep Oncol 10: 368-371, 2017.

8. Kubo T, Sugawara T, Shinkawa T, et al. Fatal fulminant hepatitis induced by combined ipilimumab and nivolumab therapy despite favorable histologic response and confirmed by autopsy in a patient with clear cell renal cell carcinoma. Immunol Med 7: 1-6, 2020.

9. West H, McCleod M, Hussein M, et al. Atezolizumab in combination with carboplatin plus nab-paclitaxel chemotherapy compared with chemotherapy alone as first-line treatment for metastatic nonsquamous non-small-cell lung cancer (IMpower130): a multicentre, randomised, open-label, phase 3 trial. Lancet Oncol 20: 924937, 2019.

10. Schmid P, Rugo HS, Adams $S$, et al. Atezolizumab plus nabpaclitaxel as first-line treatment for unresectable, locally advanced or metastatic triple-negative breast cancer (IMpassion130): updated efficacy results from a randomised, double-blind, placebocontrolled, phase 3 trial. Lancet Oncol 21: 44-59, 2020.

11. Finn RS, Qin S, Ikeda M, et al. Atezolizumab plus bevacizumab in unresectable hepatocellular carcinoma. $\mathrm{N}$ Engl $\mathrm{J}$ Med 382: 1894-1905, 2020.

12. Ribero D, Wang H, Donadon M, et al. Bevacizumab improves pathologic response and protects against hepatic injury in patients treated with oxaliplatin-based chemotherapy for colorectal liver metastases. Cancer 110: 2761-2767, 2007.

13. Fang P, Hu JH, Cheng ZG, Liu ZF, Wang JL, Jiao SC. Efficacy and safety of bevacizumab for the treatment of advanced hepatocellular carcinoma: a systematic review of phase II trials. PLoS One 7: e49717, 2012.

14. Hegde PS, Wallin JJ, Mancao C. Predictive markers of anti-VEGF and emerging role of angiogenesis inhibitors as immunotherapeutics. Semin Cancer Biol 52: 117-124, 2018.

15. Riveiro-Barciela M, Barreira-Díaz A, Vidal-González J, et al. Immune-related hepatitis related to checkpoint inhibitors: clinical and prognostic factors. Liver Int 40: 1906-1916, 2020.

16. Karamchandani DM, Chetty R. Immune checkpoint inhibitorinduced gastrointestinal and hepatic injury: pathologists' perspective. J Clin Pathol 71: 665-671, 2018.

17. De Martin E, Michot JM, Papouin B, et al. Characterization of liver injury induced by cancer immunotherapy using immune checkpoint inhibitors. J Hepatol 68: 1181-1190, 2018.

18. Zen Y, Yeh MM. Hepatotoxicity of immune checkpoint inhibitors: a histology study of seven cases in comparison with autoimmune hepatitis and idiosyncratic drug-induced liver injury. Mod Pathol 31: 965-973, 2018.

19. Koya Y, Shibata M, Shinohara N, et al. Secondary sclerosing cholangitis with hemobilia induced by pembrolizumab: case report and review of published work. Hepatol Res 49: 950-956, 2019.

20. Sangro B, Chan SL, Meyer T, Reig M, El-Khoueiry A, Galle PR. Diagnosis and management of toxicities of immune checkpoint inhibitors in hepatocellular carcinoma. J Hepatol 72: 320-341, 2020.

21. Kennedy LB, Salama AKS. A review of immune-mediated adverse events in melanoma. Oncol Ther 7: 101-120, 2019.

22. Trivedi PJ, Hubscher SG, Heneghan M, Gleeson D, Hirschfield GM. Grand round: autoimmune hepatitis. J Hepatol 70: 773-784, 2019.

23. Imoto K, Kohjima M, Hioki T, et al. Clinical features of liver injury induced by immune checkpoint inhibitors in Japanese patients. Can J Gastroenterol Hepatol 2019: 6391712, 2019.

The Internal Medicine is an Open Access journal distributed under the Creative Commons Attribution-NonCommercial-NoDerivatives 4.0 International License. To view the details of this license, please visit (https://creativecommons.org/licenses/ by-nc-nd/4.0/).

(C) 2021 The Japanese Society of Internal Medicine Intern Med 60: 1847-1853, 2021 